la revue La revue pour l'histoire du CNRS

POUR LHISTOIRE DU CNRS $24 \mid 2009$

Soixante-dixième anniversaire du CNRS

\title{
Travail, un monde en mutation
}

\section{Philippe Askenazy}

\section{OpenEdition}

Journals

Édition électronique

URL : https://journals.openedition.org/histoire-cnrs/9092

DOI : 10.4000/histoire-cnrs.9092

ISSN : 1955-2408

Éditeur

CNRS Éditions

Édition imprimée

Date de publication : 5 octobre 2009

ISSN : 1298-9800

Référence électronique

Philippe Askenazy, «Travail, un monde en mutation », La revue pour l'histoire du CNRS [En ligne], 24 I

2009, mis en ligne le 05 octobre 2009, consulté le 20 mai 2021. URL : http://journals.openedition.org/ histoire-cnrs/9092 ; DOI : https://doi.org/10.4000/histoire-cnrs.9092

Ce document a été généré automatiquement le 20 mai 2021.

Comité pour l'histoire du CNRS 


\title{
Travail, un monde en mutation
}

\author{
Philippe Askenazy
}

1 La première intervient après la seconde guerre mondiale avec la parcellisation des tâches en milieu actif. Il s'agit là d'une rupture dans l'organisation des tâches au travail. Les prémices de cette mutation apparaissaient après la première guerre mondiale mais elle prend toute son ampleur à partir de 1945. La France connaît une période de forte industrialisation jusqu'aux débuts des années 1970.

2 Après les différents chocs pétroliers, notamment celui de 1973, le tertiaire va prendre le pas sur l'industriel jusqu'au milieu des années 1990. Cette nouvelle mutation est perçue du côté des travailleurs comme une «bonne nouvelle». Mais les chercheurs vont se rendre compte au fil de leurs études que le phénomène est plus complexe qu'il n'y paraît. Il s'agit non pas d'une bonne nouvelle mais de ce que les chercheurs ont appelé l'intensification du travail qui touche à la fois l'entreprise et les services, c'est-àdire qu'on exige du travailleur une mobilisation de toutes ses capacités. Dans cette phase, le travail industriel est transposé au tertiaire. Les chercheurs ont montré que le tertiaire s'industrialisait à partir des années 1980. Les éléments physiques et cognitifs de chaque salarié sont pris en compte : dès lors, deux formes de contrainte s'imposent dans le tertiaire.

3 Selon Philippe Askenazy, économiste, directeur de recherche au CNRS, la question des conditions de travail se pose vraiment et prend toute sa légitimité : «Si nous regardons les travaux de recherche dans les années 1970, à l'époque, nous n'avions pas du tout de vision sur la question. Les chercheurs n'ont pris conscience de cette complexité qu'au milieu des années 1980 [...] En économie, il existe un courant de pensée qui s'est concentré autour de l'école de la régulation. Se posait la question entre les modes de production et la société elle-même. Robert Boyer, directeur de recherche au CNRS ${ }^{1}$ est le père de cette école de pensée dont les études se sont étendues aux pays voisins ", explique-t-il.

4 Alors qu'on observait une tertiarisation croissante de la société parallèlement à une industrialisation du tertiaire, les changements au sein des entreprises ne s'opéraient pas au même moment dans tous les pays. Si on prend l'exemple de ce que les chercheurs ont appelé la méthode japonaise, c'est-à-dire donner une certaine autonomie au travailleur tout en le contrôlant, il s'agit là d'une nouvelle manière de 
penser l'organisation du travail aussi bien dans l'industrie que dans le tertiaire. Les chercheurs comme Michel Freyssenet, Patrick Fridenson et Robert Boyer ont mis en évidence cette particularité japonaise. Grâce aux travaux de recherches du Gerpisa ${ }^{2}$, ce réseau français de chercheurs interdisciplinaires à l'origine est devenu réseau international en 1992 avec le premier programme de recherche (1993-96) sur "l'émergence de nouveaux modèles industriels", coordonné par R. Boyer, M. Freyssenet et animé par un comité international. Ces études ont permis à ces méthodes japonaises de s'adapter et de s'implanter dans les entreprises américaines lorsque les Japonais dans les années 1980 ont créé et importé leurs méthodes de travail aux ÉtatsUnis. Dans le même temps, les chercheurs s'organisent pour étudier à la fois ces modes de transformation outre-Atlantique et ce qui se passe en Europe avec les modes de production.

Dès 1990, une seconde vague d'études dans le domaine de la microéconomie va voir le jour. Économistes, sociologues, ergonomes, psychologues du travail se sont posé la question: quelles incidences ont ces mutations sur les conditions de travail ? Chacun dans sa discipline respective va tirer la sonnette d'alarme mais les premiers à travailler ensemble seront les ergonomes et les sociologues. Michel Gollac et Serge Volkoff ${ }^{3}$, créateurs des enquêtes sur les conditions de travail, ont démontré par les chiffres ce phénomène d'intensification avec un durcissement du monde du travail.

6 Au milieu des années 1990, P. Askenazy commence à travailler sur ces mêmes questions. Il est d'ailleurs l'un des rares chercheurs à s'intéresser aux conditions de travail et à la nouvelle économie. Fin 1990, un dialogue s'instaure entre chercheurs de diverses branches. Chacun comprend à son niveau que le travailleur doit être pris dans sa globalité: dans sa sphère psychologique, physique, sociale et à travers sa performance économique. Le CNRS a joué le rôle de plaque tournante dans ces recherches. Il a permis qu'un faisceau de compréhension existe. Selon P. Askenazy, le CNRS offre une certaine liberté aux chercheurs et notamment aux jeunes: "Nous n'avons pas forcément de gros moyens financiers pour nos recherches. Mais le CNRS nous donne la possibilité de faire des études avancées sur des sujets originaux. Cette liberté et l'interdisciplinarité en son sein permettent l'innovation dans la recherche. Ceci nous distingue de l'université, plus propice à la progression des recherches sur des voies déjà ouvertes, et qui souffre d'un cloisonnement disciplinaire", souligne-t-il. Ces études ont apporté des éléments de compréhension sur les modes de fonctionnement des entreprises françaises mais elles n'ont pas eu vraiment d'impact en comparaison avec leurs homologues américains. Les chercheurs français étaient très présents à la fin des années 1960 et au début des années 1970 pour comprendre le taylorisme, son implication dans le travail et sa crise. Puis la tertiarisation a fait oublier la question du travail mais aussi les chercheurs dans ce domaine.

7 Depuis 2004, il y a eu une prise de conscience des politiques et les chercheurs sont à nouveau sollicités. La crise financière internationale actuelle, d'une certaine manière tout aussi grave que celle de 1929, ouvre un nouveau champ de recherche. Pour P. Askenazy, l'histoire du monde génère de nouveaux problèmes économiques et selon lui, on attend trop aujourd'hui du chercheur : "Celui-ci peut apporter un éclairage mais il n'a pas la solution. Cela montre aussi peut-être que nous nous sommes rapprochés des thèmes qui intéressaient vraiment la société. On s'est posé la question : quel impact peut avoir ma recherche sur la société? On a des clefs pour comprendre les différents changements 4 . Cependant, je suis incapable de vous dire ce que sera le métier d'un vendeur de la Fnac dans cinq ans", conclut-il. 
Rayonnement d'un chef de rayon

Le métier de chef de rayon n'est apparu qu'au début des années 1970. Jusqu'à la fin des années 1980, le chef de rayon encadre, gère les stocks et les commandes et occupe un véritable poste de manager. Puis, au début des années 1990, les nouvelles technologies font leur entrée dans les entreprises, bien après les États-Unis (milieu des années 1980). Dès lors, le métier de chef de rayon se transforme: il s'affranchit de la gestion des stocks alors que, simultanément à cette libération, le nombre des références, des codes barres se multiplie. En dehors de son métier de manager et de conseil auprès des clients, il remplit les rayons et peut même faire de l'ensachage aux heures de grande affluence aux caisses. Cette profession a donc connu de profondes mutations, devenant à la fois intellectuelle et physique, selon un processus que les chercheurs ont appelé intensification du travail. Actuellement, un chef de rayon peut travailler 60 heures par semaine, alors que dans les années 1980, il en totalisait plutôt 40 .

\section{NOTES}

1. Robert Boyer a été membre de l'unité mixte de recherche Paris- Jourdan sciences économiques (PSE) jusqu'à son départ à la retraite en 2008.

2. Groupe d'études et de recherches permanent sur l'industrie et les salariés de l'automobile, créé par M. Freyssenet (sociologue au CNRS) et P. Fridenson (historien à l'EHESS).

3. M. Gollac et S. Volkoff, chercheurs à l'Insee. Les conditions de travail aujourd'hui. La Découverte, coll. Repères, 2007.

4. P. Askenazy et D. Cohen (sous la dir. de). 27 questions d'économie contemporaine. Albin Michel, coll. Économiques 1, 2008.

\section{RÉSUMÉS}

Depuis l'après-guerre, les chercheurs du CNRS et d'ailleurs ont pu observer trois grandes mutations dans le monde du travail: la conception tayloriste, l'industrialisation et la tertiarisation du travail avec une industrialisation qui pénètre le tertiaire. Les explications de Philippe Askenazy.

\section{AUTEUR}

\section{PHILIPPE ASKENAZY}

Philippe Askenazy, directeur de recherche au CNRS est membre de l'unité mixte de recherche Paris- Jourdan sciences économiques (PSE). 